\title{
Erratum to: Modeling Seasonal Rabies Epidemics in China
}

\author{
Juan Zhang • Zhen Jin • Gui-Quan Sun • \\ Xiang-Dong Sun · Shigui Ruan
}

Published online: 11 December 2012

(C) Society for Mathematical Biology 2012

\section{Erratum to: Bull Math Biol (2012) 74:1226-1251 DOI 10.1007/s11538-012-9720-6}

There were a few errors in the paper which are corrected as follows.

1. Page 1231 , line 4 from the bottom, the sentence after "which is defined as $z_{0}$ such that" should be revised as " $\rho\left(W\left(\omega, 0, z_{0}\right)\right)=1$ which can be calculated and detailed computations are given in the Appendix".

2. Page 1239 , line 6 from the bottom, " 226,920 " should be replaced by " 226,890 ".

3. Page 1242, Fig. 7 should be revised as follows:

The online version of the original article can be found under doi:10.1007/s11538-012-9720-6.

J. Zhang · Z. Jin · G.-Q. Sun

Department of Mathematics, North University of China, Taiyuan, Shan’xi 030051,

People's Republic of China

X.-D. Sun

The Laboratory of Animal Epidemiological Surveillance, China Animal Health and Epidemiology

Center, Qingdao, Shandong 266032, People's Republic of China

S. Ruan (凶)

Department of Mathematics, University of Miami, Coral Gables, FL 33124-4250, USA

e-mail: ruan@math.miami.edu 
(a)

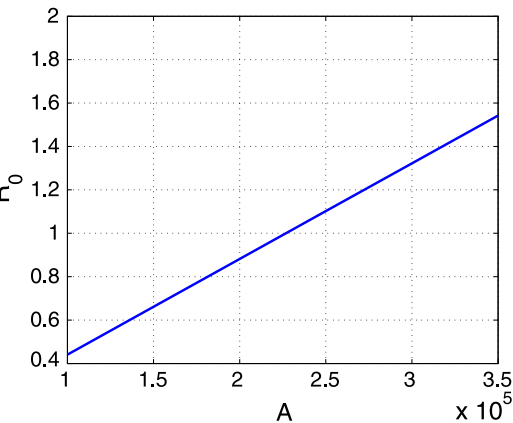

(c)

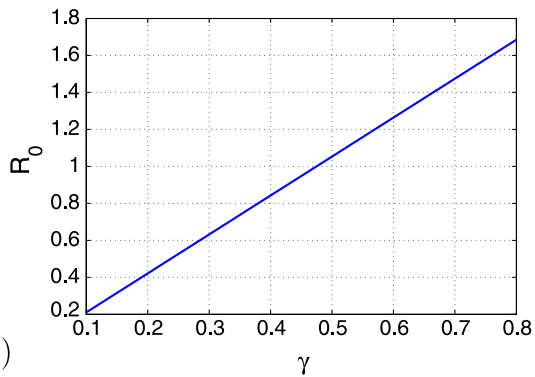

(b)

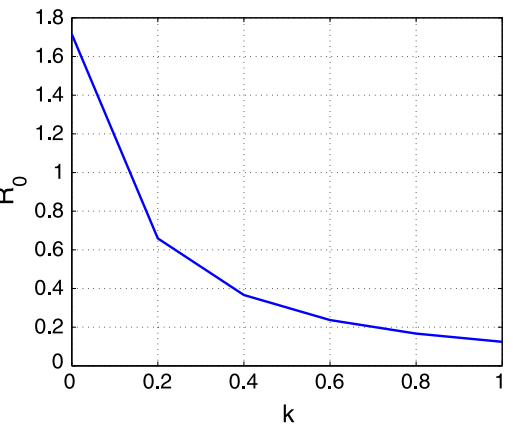

(d)

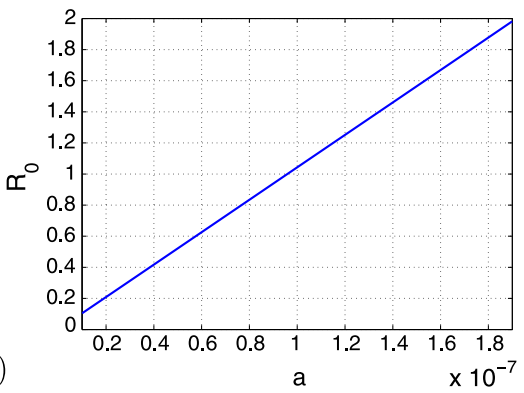

Fig. 7 The influence of parameters on $R_{0}$. (a) versus $A$; (b) versus $k$; (c) versus $\gamma ;(\mathbf{d})$ versus $a$. Other parameter values in Table 1 do not change

4. In the Appendix, page 1248, all content after "Using (ii) in Theorem 2.1 in Wang and Zhao (2008), we derive" should be replaced by the following paragraphs:

$$
\begin{aligned}
G(t) & =\frac{F(t)}{z}-V(t) \\
& =\left(\begin{array}{cccc}
-(m+\sigma+k) & 0 & \frac{\beta(t) \hat{S}}{z} & 0 \\
0 & -\left(m_{1}+\sigma_{1}+k_{1}\right) & \frac{\beta_{1}(t) \hat{S_{1}}}{z} & 0 \\
\sigma \gamma & 0 & -(m+\mu) & 0 \\
0 & \sigma_{1} \gamma_{1} & 0 & -\left(m_{1}+\mu_{1}\right)
\end{array}\right),
\end{aligned}
$$

where $\beta(t)=a\left[1+b \sin \left(\frac{\pi}{6} t+5.5\right)\right]$ and $\beta_{1}(t)=a_{1}\left[1+b_{1} \sin \left(\frac{\pi}{6} t+5.5\right)\right]$. We calculate the monodromy matrix of the system

$$
\frac{d x}{d t}=G(t) x
$$

By observing the matrix $G(t)$, we can see that $x_{1}(t)$ and $x_{3}(t)$ are independent of $x_{2}(t)$ and $x_{4}(t)$ and can be solved directly. To solve $x_{2}(t)$ and $x_{4}(t)$, consider the system

$$
\frac{d x_{2}(t)}{d t}=-\left(m_{1}+\sigma_{1}+k_{1}\right) x_{2}(t)+\frac{\beta_{1}(t) \hat{S}_{1}}{z} x_{3}(t),
$$




$$
\frac{d x_{4}(t)}{d t}=-\left(m_{1}+\mu_{1}\right) x_{4}(t)+\sigma_{1} \gamma_{1} x_{2}(t)
$$

From Eq. (18), we have

$$
x_{2}(t)=e^{-\left(m_{1}+\sigma_{1}+k_{1}\right) t}\left[c_{2}+\int_{0}^{t} \frac{\beta_{1}(s) \hat{S}_{1}}{z} x_{3}(s) e^{\left(m_{1}+\sigma_{1}+k_{1}\right) s} d s\right] \text {, }
$$

where $c_{2}$ is an arbitrary constant. Combining Eqs. (19) and (20), we have

$$
x_{4}(t)=e^{-\left(m_{1}+\mu_{1}\right) t}\left[c_{4}+\int_{0}^{t} \sigma_{1} \gamma_{1} x_{2}(s) e^{\left(m_{1}+\mu_{1}\right) s} d s\right],
$$

where $c_{4}$ is an arbitrary constant. We can verify that $\left(0, c_{2} e^{-\left(m_{1}+\sigma_{1}+k_{1}\right) t}, 0\right.$, $\left.\frac{\sigma_{1} \gamma_{1} c_{2}}{\mu_{1}-\sigma_{1}-k_{1}}\left[e^{-\left(m_{1}+\sigma_{1}+k_{1}\right) t}-e^{-\left(m_{1}+\mu_{1}\right) t}\right]\right)$ and $\left(0,0,0, c_{4} e^{-\left(m_{1}+\mu_{1}\right) t}\right)$ are two linearly independent solutions of system (17). Thus, by the necessary condition that the monodromy matrix evaluated at $T=0$ must be the identity matrix, we firstly give the form of the monodromy matrix of system (17):

$$
\Phi_{\frac{F}{z}-V}(T, z)=W(T, 0, z)=\left(\begin{array}{cccc}
a_{11} & 0 & a_{13} & 0 \\
a_{21} & a_{22} & a_{23} & 0 \\
a_{31} & 0 & a_{33} & 0 \\
a_{41} & a_{42} & a_{43} & a_{44}
\end{array}\right),
$$

where

$$
\begin{aligned}
& a_{22}=e^{-\left(m_{1}+\sigma_{1}+k_{1}\right) T}, \\
& a_{42}=\frac{\sigma_{1} \gamma_{1}}{\mu_{1}-\sigma_{1}-k_{1}} e^{-\left(m_{1}+\mu_{1}\right) T}\left[e^{\left(\mu_{1}-\sigma_{1}-k_{1}\right) T}-1\right], \\
& a_{44}=e^{-\left(m_{1}+\mu_{1}\right) T} .
\end{aligned}
$$

Note that $a_{22}$ and $a_{44}$ are two eigenvalues of the monodromy matrix and are irrelevant to $z$. Hence, it suffices to estimate the monodromy matrix $\Phi_{1}(T, z)$ of the following system

$$
\left(\begin{array}{c}
\frac{d x_{1}(t)}{d t} \\
\frac{d x_{3}(t)}{d t}
\end{array}\right)=\left(\begin{array}{cc}
-(m+\sigma+k) & \frac{a\left[1+b \sin \left(\frac{\pi}{6} t+5.5\right)\right] \hat{S}}{z} \\
\sigma \gamma & -(m+\mu)
\end{array}\right)\left(\begin{array}{l}
x_{1}(t) \\
x_{3}(t)
\end{array}\right) .
$$

and find $z_{0}$ such that $\rho\left(\Phi_{1}\left(T, z_{0}\right)\right)=1$, where $\hat{S}$ is defined in Sect. 4 .

Since the above system is linear and periodic, we can apply the shifted Chebyshev polynomials method presented in Sinha and Wu (1991). Following Fox and Parker (1968) and Luke (1969), the shifted Chebyshev polynomials of the first kind are defined on the interval $[0,1]$ by $T_{0}^{*}(t)=1, T_{1}^{*}(t)=2 t-1$ and the recursion formula

$$
T_{r+1}^{*}(t)=2(2 t-1) T_{r}^{*}(t)-T_{r-1}^{*}(t) .
$$


By the definition, we can see that the shifted Chebyshev polynomials are orthogonal:

$$
\int_{0}^{1} T_{r}^{*}(t) T_{k}^{*}(t) \omega(t) d t= \begin{cases}0 & r \neq k \\ \frac{\pi}{2} & r=k \neq 0 \\ \pi & r=k=0\end{cases}
$$

where $\omega(t)=\left(t-t^{2}\right)^{-1 / 2}$ is the weight function given by Sinha and Butcher (1995). Assume that $f(t)$ is a continuous scalar function and can be expanded in shifted Chebyshev polynomials:

$$
f(t)=\sum_{i=0}^{\infty} p_{i} T_{i}^{*}(t), \quad 0 \leq t \leq 1 .
$$

The coefficients $p_{i}$ are given by

$$
p_{i}=\frac{1}{\delta} \int_{0}^{1} \omega(\tau) f(\tau) T_{i}^{*}(\tau) d \tau, \quad i=0,1,2,3, \ldots,
$$

and

$$
\delta= \begin{cases}\frac{\pi}{2} & i \neq 0 \\ \pi & i=0\end{cases}
$$

Noticing the fact that the shifted Chebyshev polynomials are defined on the interval $[0,1]$, we can use a linear transformation $t=12 t^{*}$ and rewrite the above system as follows:

$$
\frac{d y}{d t^{*}}=\left[A\left(t^{*}\right)\right] y,
$$

where $y=\left(y_{1}\left(t^{*}\right), y_{2}\left(t^{*}\right)\right)^{T}=\left(x_{1}\left(12 t^{*}\right), x_{3}\left(12 t^{*}\right)\right)^{T}$ and

$$
\left[A\left(t^{*}\right)\right]=12\left(\begin{array}{cc}
-(m+\sigma+k) & \frac{a\left(1+b \sin \left(2 \pi t^{*}+5.5\right) \hat{S}\right.}{z} \\
\sigma \gamma & -(m+\mu)
\end{array}\right),
$$

which is a $2 \times 2$ matrix of principal period 1 . Denote $A\left(t^{*}\right)=A_{0}+A_{1}\left(t^{*}\right)$, where

$$
\begin{aligned}
& A_{0}=12\left(\begin{array}{cc}
-(m+\sigma+k) & \frac{a \hat{S}}{z} \\
\sigma \gamma & -(m+\mu)
\end{array}\right), \\
& A_{1}\left(t^{*}\right)=12\left(\begin{array}{ll}
0 & \frac{a b\left(\sin \left(2 \pi t^{*}+5.5\right)\right) \hat{S}}{z} \\
0 & 0
\end{array}\right) .
\end{aligned}
$$

Let

$$
C_{A}=12\left(\begin{array}{cc}
0 & \frac{a b \hat{S}}{z} \\
0 & 0
\end{array}\right)
$$


which is the coefficient matrix of $A_{1}\left(t^{*}\right)$. The solution vector $y\left(t^{*}\right)$ and the function $\sin \left(2 \pi t^{*}+5.5\right)$ of system (25) can be expanded in terms of the shifted Chebyshev polynomials on the interval $[0,1]$ as follows. Here, we take 15 terms of the shifted Chebyshev polynomials.

$$
\begin{gathered}
y_{j}\left(t^{*}\right) \approx \sum_{i=0}^{14} b_{i}^{j} T_{i}^{*}\left(t^{*}\right) \equiv \mathbf{T}^{* T}\left(t^{*}\right) \mathbf{b}^{j}, \quad j=1,2 . \\
\sin \left(2 \pi t^{*}+5.5\right) \approx \sum_{i=0}^{14} d_{i} T_{i}^{*}\left(t^{*}\right) \equiv \mathbf{T}^{* T}\left(t^{*}\right) \mathbf{d},
\end{gathered}
$$

where

$$
\begin{aligned}
\mathbf{b}^{j}= & \left\{b_{0}^{j}, b_{1}^{j}, b_{2}^{j}, \ldots, b_{14}^{j}\right\}^{T}, \quad j=1,2 . \\
\mathbf{d}= & \left\{d_{0}, d_{1}, d_{2}, \ldots, d_{14}\right\}^{T} \\
= & \{-0.2147,-0.4034,-0.6850,0.4726,0.2137,-0.0739, \\
& -0.0205,0.0048,9.8228 \times 10^{-4},-1.7717 \times 10^{-4},-2.8356 \times 10^{-5}, \\
& \left.4.1476 \times 10^{-6}, 5.1665 \times 10^{-7}, 1.0990 \times 10^{-7}, 9.2387 \times 10^{-7}\right\},
\end{aligned}
$$

and

$$
\mathbf{T}^{* T}\left(t^{*}\right)=\left\{T_{0}^{*}\left(t^{*}\right), T_{1}^{*}\left(t^{*}\right), \ldots, T_{14}^{*}\left(t^{*}\right)\right\} .
$$

For convenience, we introduce some notation. Let

$$
\hat{\mathbf{T}}\left(t^{*}\right)=\mathbf{I} \otimes \mathbf{T}^{* T}\left(t^{*}\right), \quad \bar{y}(0)=y(0) \otimes(1,0, \ldots, 0)^{T},
$$

where $\otimes$ represents the Kronecker product, $\mathbf{I}$ is a $2 \times 2$ identity matrix, and $y(0)$ is the initial condition.

According to the method proposed by Sinha and Wu (1991), the monodromy matrix is given by

$$
\Phi_{1}(1, z)=\hat{\mathbf{T}}(1) \bar{B}
$$

where $\Phi_{1}(0, z)=I . \bar{B}=\left[\bar{b}_{1}, \bar{b}_{2}\right]$ can be obtained by

$$
[I-Z] \bar{b}=\bar{y}(0),
$$

with initial conditions $y_{1}(0)=(1,0), y_{2}(0)=(0,1)$. Z is a $30 \times 30$ constant matrix defined by

$$
Z=A_{0} \otimes \bar{G}^{T}+C_{A} \otimes \bar{G}^{T} \bar{Q}=12\left(\begin{array}{cc}
-(m+\sigma+k) \bar{G}^{T} & \frac{a \hat{S}}{z} \bar{G}^{T}(I+b Q) \\
\sigma \gamma \bar{G}^{T} & -(m+\mu) \bar{G}^{T}
\end{array}\right),
$$

where $\bar{G}$ the $15 \times 15$ integration operational matrix, $Q$ the $15 \times 15$ product operation matrix, given respectively by 


$$
\begin{aligned}
\bar{G} & =\left(\begin{array}{cccccc}
\frac{1}{2} & \frac{1}{2} & 0 & \ldots & 0 & 0 \\
-\frac{1}{8} & 0 & \frac{1}{8} & \ldots & 0 & 0 \\
-\frac{1}{6} & -\frac{1}{4} & 0 & \ldots & 0 & 0 \\
\cdots & \ldots & \ldots & \ldots & \ldots & \ldots \\
\frac{1}{336} & 0 & 0 & \ldots & 0 & \frac{1}{56} \\
-\frac{1}{390} & 0 & 0 & \ldots & -\frac{1}{52} & 0
\end{array}\right), \\
Q & =\left(\begin{array}{cccccc}
d_{0} & \frac{d_{1}}{2} & \frac{d_{2}}{2} & \ldots & \frac{d_{13}}{2} & \frac{d_{14}}{2} \\
d_{1} & d_{0}+\frac{d_{2}}{2} & \frac{d_{1}+d_{3}}{2} & \ldots & \frac{d_{12}+d_{14}}{2} & \frac{d_{13}+d_{15}}{2} \\
d_{2} & \frac{d_{1}+d_{3}}{2} & d_{0}+\frac{d_{4}}{2} & \ldots & \frac{d_{11}+d_{15}}{2} & \frac{d_{12}+d_{16}}{2} \\
\cdots & \cdots & \ldots & \ldots & \cdots & \ldots \\
d_{13} & \frac{d_{12}+d_{14}}{2} & \frac{d_{11}+d_{15}}{2} & \ldots & d_{0}+\frac{d_{26}}{2} & \frac{d_{1}+d_{27}}{2} \\
d_{14} & \frac{d_{13}+d_{15}}{2} & \frac{d_{12}+d_{16}}{2} & \ldots & \frac{d_{1}+d_{27}}{2} & d_{0}+\frac{d_{28}}{2}
\end{array}\right),
\end{aligned}
$$

in which $d_{15}=\cdots=d_{28}=0$. After obtaining the monodromy matrix, we can find $z_{0}$, that is, the basic reproduction number satisfying $\rho\left(\Phi_{1}\left(1, z_{0}\right)\right)=1$.

Using Matlab, we obtain $z_{0}=1.0321$. Note that if we discrete the system (18)-(19) with the identity matrix as the initial state and solve $z_{0}$ directly, then $z_{0}=1.032$. We can see that these two values are very close.

Acknowledgements The authors would like to thank Dr. Nicolas Bacaëar for pointing out the error in deriving the basic reproduction number in the Appendix and Dr. Xiao-Qiang Zhao, Dr. Wendi Wang, and Dr. Daozhou Gao for helpful comments and suggestions.

\section{References}

Fox, L., \& Parker, I. B. (1968). Chebyshev Polynomials in Numerical Analysis. Oxford: Oxford University Press.

Luke, Y. L. (1969). The Special Functions and Their Approximations. New York: Academic Press.

Sinha, S. C., \& Butcher, E. A. (1995). Solution and stability of a set of $p$ th order linear differential equations with periodic coefficients via Chebyshev polynomials. Math. Problems Engineer, 2, 165-190.

Sinha, S. C., \& Wu, D.-H. (1991). An efficient computational scheme for the analysis of periodic systems. J. Sound Vib., 15(1), 91-117. 